\title{
Vorwort zur 2., vollständig neu bearbeiteten und erweiterten Auflage
}

\author{
1. Der Ausgangspunkt \\ 2. Leitlinien der Konzeption \\ 3. Leitlinien der Gliederung \\ 4. Einzelerläuterungen zur Gliederung \\ 5. Zur Geschichte der zweiten Auflage und Danksagungen
}

\section{Der Ausgangspunkt}

Die erste Auflage des Handbuches Sprachgeschichte hat in erstaunlich kurzer Zeit ihren Weg sowohl in die wissenschaftlichen und die großen öffentlichen Bibliotheken wie in die Arbeitszimmer der Sprachhistoriker und anderer an Sprachgeschichte und Sprachgeschichtsschreibung Interessierter gefunden. Das Handbuch ist damit bereits wenige Jahre nach seinem Erscheinen zum anerkannten Hilfsmittel akademischer Lehre und akademischen Lernens geworden; in beachtlichem Ausmaß hat es forschungsleitende Anstöße gegeben wie interdisziplinäre Beachtung - etwa in der Romanistik, Anglistik und Indogermanistik — gefunden. Diese Einschätzung vermitteln auch die zahlreichen, zum Teil im Umfang von Zeitschriftenartikeln erschienenen Rezensionen. Sofern Kritik geäußert wird, bezieht sich diese auf Einzelartikel oder bestimmte Einzelteile der Konzeption, nicht aber auf deren Gesamtanlage.

Dem Verlag und den Herausgebern (so hier, teilweise auch im folgenden zusammenfassend für: die Herausgeberin und die Herausgeber) sind folgende, in Umfang und Inhalt über bloße Anzeigen hinausgehende Rezensionen bekannt geworden:

Dallapiazza, Michael, Bestandsaufnahme der deutschen Sprache. In: DAAD Letter 1, 1986.

Sowinski, Bernhard in: Germanistik 26, 1985/1, 42-43 [für Halbband 1] und 27, 1986/3, 502 [für Halbband 2].

Kyes, Robert L. in: Michigan Germanic Studies 12/1, 1986, 71-73.

Baldinger, Kurt in: Zeitschrift für romanische Philologie 103 (H. 5/6), 1987, 511—518.

Bauer, Erika in: Leuvense Bijdragen 76, 1987, 525-531.

Ebert, Robert Peter in: Beiträge zur Geschichte der deutschen Sprache und Literatur (Tübingen) 109, 1987, 274-288.

Görlach, Manfred in: Word 36, 1985, 266-269 [für Halbband 1] und 38, 1987, 209-216 [für Halbband 2]. Hundsnurscher, Franz in: Anzeiger für deutsches Altertum und deutsche Literatur 98/4, 1987, 144 -171. Janota, Johannes in: Literature, Music, Fine Arts 20 (Nr. 2), 1987, 101-102.

Keller, R. E. in: Zeitschrift für Deutsche Philologie 106/1, 1987, 146-154.

Olt, Reinhard, Vom Indogermanischen zur Mediensprache. Ein Handbuch zur Geschichte des Deutschen in zwei Bänden. In: Frankfurter Allgemeine Zeitung Nr. 181/1987, 8. August, S. 6.

Wolff, Gerhart in: Wirkendes Wort 37, 1987, 176-179.

Lee, Dok-Ho, Was ist Sprachgeschichte? [Übersetzt aus dem Koreanischen]. In: Germanistik [koreanisch] 1994, 1-42.

Olt, Reinhard in: Muttersprache 1988/2, 164-166.

Seebold, Elmar in: Indogermanische Forschungen 93, 1988, 274-280. 
Fleischer, Wolfgang in: Zeitschrift für Phonetik, Sprachwissenschaft und Kommunikationsforschung 42, 1989, 260-264.

Penzl, Herbert in: Language 65/3, 1989, 637-641.

Voorwinden, Norbert in: Modern Language Review 82/4, 1987, 1012-1015.

Brandt, Gisela in: Zeitschrift für Germanistik 6, 1990, 730—733.

Heringer, Hans Jürgen in: Sprache und Literatur 66, 1990, 110_-111.

\section{Leitlinien der Konzeption}

Die Herausgeber haben anerkennende und kritische Aussagen der Rezensionen und sonstiger Bezugnahmen auf das Handbuch systematisch gesammelt und gewichtet. Sie haben sich außerdem bemüht, die seit der Mitte der achtziger Jahre erschienene Literatur zur Sprachgeschichte des Deutschen sowie zur Theorie und Methode der Sprachgeschichtsschreibung generell zu registrieren; dabei wurde auch der geschichtlichen Erforschung der Nachbarsprachen konstante Aufmerksamkeit gewidmet. Einige der in der ersten Auflage des Handbuches zwar angelegte, aber noch nicht hinreichend entfaltete Ideen konnten auf diese Weise stärker gewichtet und eine Reihe neuer Ideen entwickelt werden. Aus all dem ergibt sich folgendes Verhältnis der zweiten zur ersten Auflage:

(1) Die Aufgabe des Handbuches (wie wir sie sehen), seine allgemeine sprachtheoretische und methodische Ausrichtung, die Grundlinien seiner Inhaltsgliederung und der Kapitelfolge, damit eine hohe Anzahl seiner Artikel(titel) blieben erhalten. Das Vorwort zur ersten Auflage wird, damit die angedeuteten programmatischen Aussagen nicht wiederholt werden müssen, hier in voller Länge (in Petitsatz) wieder abgedruckt. Stichwortartig seien zusätzlich die Schwerpunkte zusammengestellt, auf die es uns in besonderer Weise ankam und weiterhin, zum Teil verstärkt, ankommt: ausgewogene Beschreibung aller hierarchischen Systemebenen der Sprache von der Phonemik/Graphemik aufwärts über die Flexions- und Wortbildungsmorphologie, die Lexik und Syntax bis hin zur Textgeschichte; vergleichbar ausführliche Berücksichtigung der Systemgeschichte des Deutschen wie der Geschichte aller seiner räumlichen, zeitlichen, sozialen, fachlichen und sonstigen gruppenbestimmten Varietäten sowie der gesprochenen und der geschriebenen Sprache; konsequente Beachtung des Zusammenhangs von Sprachgeschichte und kulturellem Funktionszusammenhang der Sprache und des Sprechens; Herausstellung des Wechselverhältnisses von Sprachgeschichte und Kulturgeschichte sowie von Sprachgeschichts- und Kulturgeschichtsschreibung; Betonung der Bezüge zwischen Sprach- und Literaturgeschichte wie der darauf bezogenen Wissenschaften; Heraushebung der Europäizität des Deutschen.

(2) Aus diesen Zielsetzungen ergaben sich einige eingreifende Veränderungen auf der Makroebene, also in der Kapitelgliederung; diese können Kapitelzusammenfassungen, Kapitelspaltungen und Hinzufügungen neuer Kapitel sein.

So wurde (als Kapitelzusammenfassung) die historische Betrachtung des alten Kapitels IV (Wissenschaftshistorische Stufen sprachgeschichtlicher Forschung II: Geschichte der Sprachgeschichtsforschung nach Beschreibungsebenen) mit der systematischen des Kapitels V (Bausteine einer Prinzipienlehre und Methodik der Beschreibung historischer Sprachstufen nach Beschreibungsebenen) zu nunmehr IV. Geschichte und Prinzipien der Sprachgeschichtsforschung nach Beschreibungsebenen verbunden. Umgekehrt erfuhren drei Kapitel eine Spaltung:

- XIV. Ergebnisse der Sprachgeschichtsforschung zu den historischen Sprachstufen VI: Das Neuhochdeutsche in seiner Entwicklung vom 17. bis zum 20. Jahrhundert wurde auf die Zeit bis zur Mitte des 20. Jahrhunderts beschränkt (als neues Kap. XIII); hinzukam demzufolge: XIV. Entwicklungstendenzen der deutschen Sprache seit der Mitte des 20. Jahrhunderts. 
- XV. Ergebnisse der Sprachgeschichtsforschung im Gesamtüberblick wurde zu nunmehr $X V$. Ergebnisse der Sprachgeschichtsforschung im Gesamtüberblick I: Pragmatische und soziologische Aspekte und XVI. Ergebnisse der Sprachgeschichtsforschung im Gesamtüberblick II: Sprachsystematische Aspekte.

- VII. Das Deutsche im Sprachkontakt wurde zu (nunmehr) XIX. Das Deutsche im Sprachenkontakt I: Systematische und soziologische Aspekte und XX. Das Deutsche im Sprachenkontakt II: Aspekte der Sprachgrenzbildung des Deutschen.

Neu hinzugefügt wurden die Kapitel VII. Aspekte einer europäischen Sprachgeschichte und XVIII. Grundlinien einer literarischen Sprachgeschichte des Deutschen.

Die Einzelerläuterungen zur Gliederung (s. u. Abschn. 4) geben über die Veränderungen und deren Begründung weitere Auskunft.

(3) Auf der Mikroebene (= Artikelebene), und zwar innerhalb nahezu aller Kapitel, erfolgten Modifikationen alter Titelformulierungen, Erweiterungen, Kürzungen und in vielen Fällen Hinzufügungen neuer Artikel; in einer Reihe von Fällen wurden Umstellungen in neue Kapitelzusammenhänge vorgenommen. Alle Artikel, auch die im Titel unveränderten, wurden einer Neuredaktion unterzogen; die Neufassung schwankt zwischen geringfügigen Nachträgen und der Formulierung eines vollständig neuen Textes. Dies letztere ist nahezu immer dann der Fall, wenn die Autorschaft gewechselt hat. Besondere Aufmerksamkeit wurde der Verzeichnung neuer Forschungsliteratur gewidmet; eine maßvolle Vermehrung von Skizzen, Tabellen und Abbildungen dient der Erhöhung des Dokumentations- und Veranschaulichungswertes des Handbuches.

Das Handbuch behält damit die Aufgabe,

- die wichtigsten Ergebnisse der bisherigen Sprachgeschichtsforschung des Deutschen zusammenfassend darzustellen,

- diese Ergebisse in ihrer jeweiligen sozialhistorischen, wissenschaftsgeschichtlichen, sprachtheoretischen und methodischen Bindung zu erhellen,

- aus der Übersicht über die Forschungsergebnisse und aus der Kenntnis ihrer wissenschaftsgeschichtlichen Bindung auf Ergebnis-, Theorie- und Methodenlücken hinzuweisen und damit neue wissenschaftliche Perspektiven anzudeuten,

- die Grundzüge einer heutigen Ansprüchen genügenden Prinzipienlehre und Methodik der Sprachgeschichtsforschung zu erarbeiten und in ihrer Problematik sowie Leistungsfähigkeit zu diskutieren,

- im Zusammenhang damit den Gegenstand der Sprachgeschichtsforschung so zu begründen, daß Sprechen und Schreiben einerseits in ihrer sozialen, arealen, situativen, literarischen und allgemein kulturgeschichtlichen Heterogenität und andererseits in ihrer Systemhaftigkeit erkannt werden,

- dies alles so zu tun, daß einer Vielzahl von Leserinnen und Lesern bei der Lektüre der drei Teilbände die Überzeugung vermittelt wird: tua res agitur, es ist Deine Geschichte, Deine Gegenwart, Deine Zukunft, letztlich Deine kulturelle Identität, die hier behandelt wird.

Mit diesen Zielen wendet sich das Handbuch auch in seiner zweiten Auflage zunächst an alle diejenigen, die sich in Forschung und Lehre mit der Geschichte der Einzelsprache Deutsch und seiner Erforschung befassen. Einzelne Kapitel des Handbuches, insbesondere die auf die gesellschaftliche Verflechtung der Sprachgeschichte sowie auf die Theorie und Methode der Sprachgeschichtsforschung bezogenen Handbuchteile, haben einen systematischen Aussagewert für die Erforschung anderer, unter vergleichbaren Aspekten behandelbarer Einzelsprachen zumindest des europäischen Raumes. Sofern das Handbuch am Beispiel des Deutschen allgemeine Gesichtspunkte der Geschichte einer Sprache behandelt, sind die Vertreter der Allgemeinen Sprachwissenschaft angesprochen.

Außer den in sprachwissenschaftlicher Forschung und Lehre Tätigen zählen aber auch alle anderen an der deutschen Kulturgeschichte (im weitesten Sinne) Interessierten zum Adressatenkreis des Handbuches. Für sie ist Sprachgeschichte relevante Nachbardisziplin; sie ist Grundwissenschaft, insofern sie Möglichkeiten und Grenzen des Textverstehens bestimmt 
und damit historische, darunter z. B. literatur-, rechts-, begriffs-, ideen-, geistes-, sozial-, theologiegeschichtliche Forschung wissenschaftlich fundiert; sie ist Hilfsdisziplin, sofern sie - wie die Namenkunde — besondere Quellenmaterialien erschließt und auswertet oder — wie die Editionsphilologie - wissenschaftlich aufbereitete Quellentexte anbietet.

Die theoretische Basisausrichtung auch der zweiten Auflage liefert die seit dem 19. Jahrhundert in wechselnden zeitgeschichtlichen Varianten vertretene kulturhistorisch orientierte Sprachforschung. Das ist diejenige Konzeption, die den Sprachgebrauch prinzipiell in Wechselbeziehung zu dem kulturellen, sowohl materiellen wie geistigen, Umfeld sieht, aus dem heraus sprachliche Äußerungen ihren Sinn erhalten und zu dessen Konstituierung und Geschichte sie ihrerseits beitragen. Es ist damit gleichzeitig diejenige Konzeption, die den Brükkenschlag zu den Erkenntnisanliegen anderer historischer Disziplinen am ehesten zu vollziehen in der Lage ist und Sprachgeschichtsforschung als Teil einer umfassenden Traditionsforschung begründet; sie leistet infolge dieser Ausrichtung einen Beitrag zu den von Schule und Kulturinstanzen seit dem vorigen Jahrhundert getragenen Bemühungen, die jeweilige zeitgenössische Konstellation der Gesellschaft als Ergebnis von Geschichte zu begreifen. Indem die kulturhistorisch orientierte Sprachforschung die Heterogenität des sprachlichen Handelns als wichtigen Gegenstand sieht, erhält der textliche Überlieferungsbestand eine herausragende Rolle für die damit empirisch begründete Methodik. Alle Konstruktionen historischer Systeme (durch welche geschichtswissenschaftlichen Einzeldisziplinen auch immer) gehen von der Überlieferung aus oder werden, wo sich deduktive Bauteile als notwendig erweisen, fortwährend auf diese zurückbezogen. Sie haben die Funktion, die zugrundegelegte empirische Basis auf eine jeweils bestimmte Weise überschaubar zu machen und spiegeln Sprachgeschichtsschreibung damit als eine interpretative Disziplin.

Dieses Konzept hat Auswirkungen auf die im Handbuch erstrebte und weitgehend realisierte Fachsprache, darunter die Fachterminologie. Wenn Sprachgeschichte ein integraler Bestandteil von Geschichte überhaupt und Beschäftigung mit Sprachgeschichte der Ausdruck des Interesses geschichtsbewußter Menschen an der Tradition ist, dann überschreiten Sprachgeschichte und Sprachgeschichtsforschung den engen Rahmen, der einem einzelnen Fach hinsichtlich seines Gegenstandes wie seiner Rezipientengruppen üblicherweise gesetzt ist; sie suchen vielmehr bewußt den weiteren Horizont des öffentlichen, auch des politischen Interesses. Fachsprache muß dann eine zur Normalsprache hin offene Variante der allgemeinen Bildungssprache und damit jedem einschlägig Interessierten verständlich sein.

\section{Leitlinien der Gliederung}

Die inhaltliche Grundgliederung der zweiten Auflage ist - entsprechend derjenigen der ersten Auflage — dadurch gekennzeichnet, daß in einem ersten Kapitelblock (I-III/IV) die generelle Verflechtung der Sprachgeschichte mit der Kulturgeschichte (so Kap. I) und der Sprachgeschichtsforschung mit dem jeweiligen gesellschaftlichen Kulturbegriff (Kap. II) sowie den jeweils zeitgenössischen Erkenntnisanliegen der Sprachgeschichtsforschung (Kap. III/IV) zum Ausdruck kommt. Dem schließt sich ein zweiter Block (Kap. IV und V) mit dem Ziel an, Grundzüge einer Prinzipienlehre der Sprachgeschichtsforschung zu entwickeln und im Zusammenhang damit zentrale theoretische und methodologische Problemfelder zu diskutieren. Die Kapitel VI bis XXI sind insgesamt der Beschreibung des Faktenmaterials gewidmet, das die germanistische Sprachgeschichtsforschung seit dem 19. Jahrhundert erarbeitet hat. Den zentralen Teil dieses Blockes bilden die Kapitel VIII bis XIV, die das eigentliche materiale Corpus der deutschen Sprachgeschichte, nämlich die historischen Sprachstufen des Deutschen vom Althochdeutschen bzw. Altniederdeutschen (Altsächsischen) an bis zum Neuhochdeutschen der Gegenwart, abhandeln. Diesem Block sind zwei die linguistische Einordnung und kulturgeschichtliche Einbettung des Deutschen betreffende Kapitel vorgeschaltet. Das erste 
von ihnen verfolgt den Zweck, seine grundlegende systematische, d. h. hier: genealogische und typologische Einordnung (Kap. VI) zu beschreiben, das zweite stellt das Deutsche entlang der Zeitlinie in den Zusammenhang mit der Geschichte der Bildungs- und der Nachbarsprachen, so daß sich relevante Aspekte einer europäischen Sprachgeschichte (Kap. VII) ergeben. Dem auf die Sprachstufen bezogenen Block folgen zwei Kapitel (XV und XVI), deren Gegenstand nur epochenübergreifend $\mathrm{zu}$ behandeln ist, und zwar ein erstes, das auf pragmatische (Kap. XV) und ein zweites, das auf sprachsystematische Aspekte (Kap. XVI) zentriert ist. Die Kapitel XVII bis XXI haben sehr unterschiedliche, bisher zum Teil in Spezialdisziplinen der Sprachgeschichtsforschung behandelte Gegenstände, nämlich die Regionalsprachgeschichte anhand ausgewählter Beispiele (Kap. XVII), die Grundlinien einer literarischen Sprachgeschichte des Deutschen (Kap. XVIII), das Deutsche im Sprachenkontakt, und zwar sowohl unter systematischen und soziologischen (Kap. XIX) wie unter arealen, vor allem auf die Sprachgrenzbildung bezüglichen Aspekten (Kap. XX), schließlich die Namengeschichte (Kap. XXI).

Alle Kapitel haben, soweit die Unterschiedlichkeit der Gegenstände dies zuläßt, gewisse Parallelen in ihrer internen Gliederung. So behandeln die auf die historischen Sprachstufen des Deutschen bezogenen Kapitel (VIII bis XIII), analog dazu auch das auf die epochenübergreifenden sprachsystematischen Aspekte bezogene Kapitel (XVI), das Sprachsystem jeweils nach seinen hierarchischen Rängen, und zwar aszendent von der Phonologie/Graphematik über die Flexionsmorphologie, Lexik, Syntax, Wortbildung bis hin zu den Texten. Für die einzelnen Sprachstufen verbinden sich damit Einzelartikel über die soziokulturellen Voraussetzungen und den Sprachraum, über die Reflexe gesprochener Sprache, über die Diagliederung. Entwicklungen, die für eine einzelne Sprachstufe spezifisch sind, werden jeweils gegen Ende des Kapitels in eigenen Artikeln dargestellt. Es hängt mit dem sprachhistorischen Kenntnisstand zusammen, daß die Anzahl dieser Artikel mit dem Fortschreiten der Zeit zunimmt und im Kapitel über das Neuhochdeutsche seit der Mitte des 20. Jahrhunderts (XIV) die Gliederung nach Rangebenen schließlich ganz ersetzt. - Weitere kapitelinterne Strukturierungsgesichtspunkte liefern die Zeitgliederung (in besonderer Weise für die Kapitel III, VII, XVIII) sowie die Raumgliederung des Deutschen (Kap. XVII) bzw. die räumliche Lage seiner Nachbarsprachen (Kap. XIX, XX).

\section{Einzelerläuterungen zur Gliederung}

Kapitel I. Deutsche Sprachgeschichte im Rahmen der Kulturgeschichte hat die Verflechtung von Sprach- und Kulturgeschichte als Gegenstand. In einem einleitenden Grundsatzartikel werden Geschichts- und Sprachgeschichtsauffassungen sowie ihre Umsetzung in praktische Forschung beschrieben und diskutiert sowie die möglichen Sichtweisen des Verhältnisses von Sprache und Kultur vorgestellt. Die folgenden Artikel schließen sich dem mit der Zielsetzung an, verschiedene Einzelaspekte der Verflechtung am Beispiel einiger für die Sprachentwicklung besonders relevanter Kulturbereiche zu erörtern. Diese sind: Gesellschaft generell, Institutionen, Kirche, Recht, Politik, Wirtschaft, Alltag, Volkskultur, Technik, Philosophie, Naturwissenschaften. Die Artikel 14 bis 19 nehmen auf Gegebenheiten Bezug, die die Text- und Überlieferungsgeschichte des Deutschen als gemeinsamen Nenner haben. Kap. I dokumentiert mit diesen Inhalten wie auch mit seiner Stellung am Anfang des gesamten Handbuches das Gewicht, das die Herausgeber der Einbettung der Sprachgeschichte in die Kulturgeschichte sowie umgekehrt der Sprachabhängigkeit und sprachlichen Erscheinungsform vieler Kulturbereiche zumessen. Die Neuaufnahme von Artikeln zur Rolle des Alltags, der Institutionen und der Technik gegenüber der ersten Auflage unterstreicht das Bestreben.

Die Thematisierung des gesellschaftlichen Verständnisses der Sprachgeschichte in einem eigenen, gegenüber der ersten Auflage ebenfalls etwas erweiterten Kapitel (II) zielt auf die 
Herausarbeitung der Tatsache, daß Sprachgeschichte im Laufe der Kulturgeschichte höchstens nach dem Selbstverständnis einiger Wissenschaftler und nur sehr zeitweilig als zweckfreie Wissenschaft betrieben wurde, daß sie vielmehr seit jeher Erkenntnisinteressen vertrat, die wie z. B. die postulierte grammatische und lexikalische Reinheit des Deutschen, seine Rückverlängerung in eine möglichst lange Vorgeschichte, seine Bindung an ein Volk - in Parallele $\mathrm{zu}$ verwandten gesellschafts- und bildungspolitischen Zielen der jeweiligen Zeit standen. Die Einzelartikel des Kapitels, darunter die beiden neu aufgenommenen (Art. 20 und 23), beschreiben diese Interessengeschichte entlang der Zeitlinie sowie hinsichtlich besonders brisanter Einzelaspekte.

Die Wissenschaftsgeschichte der Historiolinguistik als Gegenstand von Kapitel III ist schon deshalb von besonderem Interesse, weil Sprachgeschichtsschreibung immer wissenschaftshistorisch bedingter Entwurf ist und ihre Ergebnisse trotz der für sie in der Regel beanspruchten Faktizität als zeitgebunden anzusehen sind. Als ebenso wesentlich erachtet es das Herausgeberteam, daß die Sprachgeschichtsforschung in ihrer fast 200 Jahre (und rund drei Jahrhunderte Vorgeschichte dazu) umfassenden Entwicklung als eigener (im heutigen Sinne) wissenschaftlicher Disziplin einen derartigen Katalog an theoretischen Annahmen, an Fragestellungen, an Methoden und Ergebnissen, überhaupt an Erfahrungs- und Kenntnisreichtum (in welchem Sinne auch immer) erarbeitet hat, daß deren Ausblendung notwendigerweise zu Einseitigkeiten und Verkürzungen in den Fragestellungen führen müßte; schließlich ist ja auch heutige geisteswissenschaftliche Forschung zeitgenössischer Entwurf und insofern der Sprachgeschichtsschreibung früherer Epochen (etwa des 19. Jahrhunderts) systematisch ähnlich, jedenfalls nicht von vorneherein überlegen. Die Aufarbeitung der Wissenschaftsgeschichte bietet mithin eine gewisse Gewähr dafür, daß man bei moderner Theorie- und Methodenentwicklung der kritischen Reflexion des eigenen Standpunktes einen hohen Stellenwert einräumt, um auch nicht hinter den Stand des Erreichten zurückzufallen (so viel Fortschrittsglaube muß erlaubt sein). - Die Einzelartikel des Kapitels behandeln entlang der Zeitlinie die bedeutendsten wissenschaftshistorischen Epochen bzw. Forschungsparadigmen; nacheinander sind dies die Zeit vom Humanismus bis zur Aufklärung (Art. 27), die von der Romantik geprägte erste Hälfte des 19. Jahrhunderts (Art. 28), die Zeit der Junggrammatiker (Art. 29), die Sprachgeographie (Art. 30), der Strukturalismus (Art. 31) und Generativismus (Art. 32) und in jüngster Zeit pragmatische Ansätze (Art. 33).

Kapitel IV. Geschichte und Prinzipien der Sprachgeschichtsforschung nach Beschreibungsebenen entwirft eine moderne Prinzipienlehre und Methodik der Sprachgeschichtsschreibung, und zwar erstens als selbst historischen, damit vorläufigen Endpunkt einer langen theoretischen und forschungspraktischen Entwicklung und zweitens als systematische Zusammenfassung heute diskutierter einzelphilologischer und allgemein linguistischer sprachtheoretischer und methodischer Anliegen. Die einzelnen Artikel des Kapitels bestehen demzufolge aus jeweils zwei ineinandergreifenden Teilen; in ihrem ersten werden pro Beschreibungsebene (Graphematik/Phonologie, Morphologie, Wortbildung, Lexikologie, Syntax, Textologie) die zentralen Theoreme und Methoden der Sprachgeschichtsschreibung (im wesentlichen seit dem 19. Jahrhundert), darunter die Sprachauffassung, die Gegenstandsbestimmung, die Ergebnistypen und die Art ihrer Präsentation, genannt und als zeitgebunden beschrieben. Mit diesem ersten Teil ergänzen die Artikel des Kapitels IV die auf wissenschaftshistorische Stufen bezogenen Darstellungen des Kapitels III unter den spezifischen Aspekten der jeweiligen Systemebene. Der zweite Teil der Artikel entwickelt darauf aufbauend unter Beachtung historischer Kontinuitäten und gleichzeitig unter Aufweisung von Brüchen die Grundzüge einer sich der Unverbindlichkeit rein historischer Registration entziehenden, modernen wissenschaftlichen Ansprüchen wie auch Erkenntnisinteressen genügenden Prinzipienlehre und Methodik der Sprachgeschichtsschreibung. Dieser Teil impliziert insofern ein wissenschaftliches Bekenntnis, als pro Beschreibungsebene z. B. zu formulieren ist, wie Geschichtlichkeit, Sprache, Sprachwandel usw. verstanden werden sollen, wie eine auf einem ausformulierten Geschichts- und Sprachverständnis beruhende Methodik operieren kann, wie das Verhältnis von 
Heterogenität und Systematik des Quellenbefundes zu gewichten ist, wie die Ergebnisse fachstilistisch gegenüber einer historisch interessierten Öffentlichkeit oder gegenüber einem engen Fachpublikum präsentiert werden sollen, von welcher Art sie sein müssen, um von Nachbardisziplinen wie z. B. der Literaturwissenschaft, Theologie, Rechtsgeschichte usw. rezipiert zu werden. - Eingelagert in das Kapitel sind einige Artikel über Gegenstände, denen wie der historischen Lexikographie (Art. 38), den Indizes und Konkordanzen (Art. 39) ein besonderes praktisches Gewicht zukommt oder denen wie der etymologischen Forschung (Art. 42) ein besonderes theoretisches Interesse entgegengegebracht werden dürfte.

Einige den Rahmen des Kapitels IV sprengende, besonders gewichtige methodische und theoretische Problemfelder werden in Kapitel V gesondert behandelt. Dazu zählen die Periodisierung der deutschen Sprachgeschichte (Art. 44), das Verhältnis von Synchronie und Diachronie (Art. 45), der Sprachwandel (Art. 46 bis 49), die Corpusbildung (Art. 50), die historische Sprachgeographie (Art. 51; 52), die Textedition (Art. 53 bis 56) und schließlich das Verstehen altdeutscher Texte (Art. 57).

Kapitel VI. Die Darstellung der genealogischen und typologischen Einordnung des Deutschen ergänzt das aus drei Artikeln bestehende Kapitel der ersten Auflage um zwei weitere Artikel, und zwar zu den germanischen Sprachen der Völkerwanderungszeit (Art. 60) und zu den typologischen Unterschieden in den Varietäten des Deutschen (Art. 62). Mit dem erstgenannten Artikel erfährt die Vorgeschichte des Deutschen, mit dem zweiten seine typologische Betrachtung eine gegenüber der ersten Auflage erheblich stärkere Gewichtung.

Dies gilt in gleicher Weise für das gesamte, neu eingefügte und besondere Anforderungen stellende Kapitel VII. Aspekte einer europäischen Sprachgeschichte. Ausgehend von der Prämisse, daß sich die Geschichte einer Einzelsprache innerhalb der kulturgeographischen Einheit Europa immer nur in wechselseitiger Beeinflussung, teils in Abgrenzung, größeren Teils in oft nicht erkannter Analogie mit derjenigen anderer Einzelsprachen vollziehen kann, werden einige der großen historischen Weichenstellungen der europäischen Kulturgeschichte auf den Erklärungswert befragt, den sie für die erstaunlichen ausdrucks- und inhaltsseitigen Gemeinsamkeiten des Deutschen mit anderen europäischen Sprachen auf allen Rängen von der Graphie und Phonologie bis hin zu den Textsorten haben können. Zu den gemeinten Weichenstellungen zählen die Sprach- und Nationenbildung in Spätantike und frühem Mittelalter (Art. 63), die Christianisierung Europas (Art. 64), das Phänomen Latinität und westeuropäische Volkssprachen (Art. 65) sowie der gesamteuropäische Prozeß der Herausbildung von Schriftsprachen (Art. 66). Diesem Artikelblock schließt sich eine Geschichte der Sprachdominanzen und ihrer Auswirkungen auf die Lehnverflechtung des Deutschen an, und zwar nach der Zeitlinie (Art. 67; 68). Zwei weitere Artikel sind den Gemeinsamkeiten und Differenzen im Wortschatz (Art. 69) bzw. in der grammatischen Struktur europäischer Sprachen gewidmet (Art. 70). - Die ersten Artikel dieses Kapitels führen zusätzlich zu denjenigen des Kapitels VI zu besonderer Beachtung der Vorgeschichte des Deutschen. - Die erwähnten besonderen Anforderungen des Kapitels ergeben sich daraus, daß die sprach- und kulturnationalen Isolierungen, denen die einzelnen philologischen und historischen Disziplinen im Laufe ihrer jeweiligen Eigenentwicklungen unterlagen, zu überwinden waren.

Die Kapitel VIII bis XIII, die die historischen Sprachstufen des Deutschen zum Gegenstand haben, bleiben in ihrer Anordnung und ihrer internen Gliederung nach Beschreibungsebenen gegenüber der ersten Auflage unverändert. Auch die Anzahl und Formulierung der zusätzlichen, nicht auf die Beschreibungsebenen bezogenen Artikel zu den einzelnen Sprachstufen erfahren nur geringfügige Änderungen. Einige Umschichtungen ergaben sich daraus, daß alle das Verhältnis von Sprach- und Literaturgeschichte betreffenden Artikel in der Neuauflage in Kapitel XVIII. Grundlinien einer literarischen Sprachgeschichte des Deutschen zusammengestellt sind. Der Inhalt der dem Mittelniederdeutschen und Frühneuhochdeutschen in der ersten Auflage gewidmeten Artikel über die Siedlungsbewegung und Sprachentwicklung im ostniederdeutschen bzw. im ostmitteldeutschen Raum (alte Art. 115 und 128) geht in der neuen Auf- 
lage in den entsprechenden sprachraumbezüglichen Artikeln des neuen Kapitels XVII. Regionalsprachgeschichte

auf.

Angesichts der Tatsache, daß die Artikel des Kapitels zum Neuhochdeutschen in der ersten Auflage des Handbuches die gesamte Zeitspanne vom 17. bis zum 20. Jahrhundert zu behandeln hatten, fehlte diesen durchgehend der Raum für ein ausführliches Eingehen auf die sprachhistorische Situation und die sprachlichen Entwicklungen nach der Mitte unseres Jahrhunderts. Der dadurch bedingte Verzicht auf eine besondere Beschreibungsintensität für die Gegenwartssprache wurde zwar durch einen umfänglichen zusammenfassenden Artikel (in der alten Auflage Nr. 151) mit dem Titel Die Entwicklung der deutschen Sprache seit 1945 und einige diesem vorgelagerte Artikel zu Spezialthemen aufgefangen; es kann aber kein Zweifel darüber bestehen, daß die erste Auflage dem Interesse, das außer den Lehrenden und Lernenden der deutschsprachigen Länder auch die Auslandsgermanistik und die an Sprache interessierte Öffentlichkeit an der jüngsten Sprachgeschichte des Deutschen hat, nicht hinreichend entgegengekommen ist. Das neue Kapitel XIV. Entwicklungstendenzen der deutschen Sprache seit der Mitte des 20. Jahrhunderts spiegelt mit 17 Artikeln das Gewicht, das der jüngsten Entwicklungsphase des Deutschen in der zweiten Handbuchauflage gewidmet wird. - Das Kapitel unterscheidet sich von den vorangehenden sprachstufenbezogenen Kapiteln dadurch, daß die Gliederung nach Hierarchieebenen durch eine Gliederung nach Gegenstandsbereichen ersetzt ist. Thematisiert werden (in teilweise mehreren Artikeln) vor allem die Existenzweisen des Deutschen, sein Gebrauch in mehreren Staaten, seine Stellung innerhalb Europas, die Sprachkritik, die feministische Linguistik, Sprache in den Medien.

Die Teilung des alten Kapitels XV. Ergebnisse der Sprachgeschichtsforschung im Gesamtüberblick in ein erstes, pragmatische und soziologische Aspekte behandelndes (Nr. XV) und ein zweites, auf systematische Aspekte bezogenes (Nr. XVI) Kapitel erwies sich aus zwei Gründen als notwendig: Erstens sollte den epochenübergreifenden Gesichtspunkten ein stärkeres Gewicht verliehen werden; zweitens sollten die im weitesten Sinne pragmatischen Gesichtspunkte der Sprachgeschichtsschreibung stringenter von denjenigen geschieden werden, die auf die Geschichte des Sprachsystems zielen.

Innerhalb des Kapitels XV wird dem Gegenstand „Stadtsprachgeschichte“ mit fünf Artikeln (Art. 160 bis 164; gegenüber nur einem in der ersten Auflage) ein besonderes Gewicht zugemessen. Anhand der Sprachgeschichte der Städte Hamburg, Berlin, Köln, Nürnberg und Wien wird die historische (diachrone) Stadtsprachenforschung als eines der bedeutendsten neueren Arbeitsgebiete der Sprachgeschichtsforschung ausdrücklich herausgestellt. Die Aufmerksamkeit gebührt dabei der Methodik der Erschließung, einer ausgewogenen Zusammenstellung und der Auswertung der Quellen, ferner der Systematisierung der Fragestellungen und der Reflexion der erzielten Ergebnisse. Bei zusammenfassender Betrachtung des Ertrages der fünf Artikel ergeben sich Bausteine einer Methodologie und Prinzipienlehre der historischen Stadtsprachenforschung. - In der Sprachgeschichtsschreibung neu ist der Artikel über Alphabetisierung (Art. 168).

Kapitel XVI knüpft an das im Vorwort zur ersten Auflage (dort S. XI) formulierte Desiderat an, analog zu einigen Artikeln der Kapitel X bis XII des Handbuchs Dialektologie wichtige systematische Aspekte der Sprachentwicklung, und zwar nach ihren Beschreibungsebenen, zu behandeln. In der (hier vorgelegten) zweiten Auflage ist das Desiderat weitgehend realisiert. Dies geschieht in einer Artikelfolge, die nacheinander Lautsystementwicklungen, graphematische, flexions- und wortbildungsmorphologische, lexikalische, phraseologische und sprichwortbezügliche sowie syntaktische Entwicklungen in ihren Grundzügen darstellt. Drei stark textlinguistisch orientierte Artikel ergänzen das Programm und dokumentieren auch von dieser Stelle aus das Gewicht, das die Herausgeber der Verbindung von Sprachsystemgeschichte und Textgeschichte (darunter Literaturgeschichte) zumessen.

Obwohl Kapitel XVII. Regionalsprachgeschichte einige Artikel der ersten Auflage (nämlich 115; 128; 161-164) fortführt, stellt es eine wesentliche Neuerung der zweiten Auflage dar. Es ist als eine heutigen Fragestellungen und Beschreibungsmöglichkeiten gerecht werdende 
Fortsetzung der berühmten rheinischen, ostmitteldeutschen, nassauischen, oberrheinischen usw. Sprachgeschichten konzipiert und hat die Geschichte der dialektalen Varianten, der in der jeweils behandelten Landschaft verwendeten Schreib- und Druckersprachen, die historische Konstanz und den Wechsel der kulturräumlichen Beeinflussungen, denen die Landschaft unterlag, umgekehrt die vom Einzelraum ausgehenden sprachlichen und literarischen Strahlungen, schließlich und vor allem den jeweiligen landschaftlichen Beitrag zur Entwicklung oder Übernahme hoch- und literatursprachlicher Varianten des Deutschen zu beschreiben. Dabei wird - so weit die Quellen dies erlauben — den gesprochenen Formen der Sprache gleiche Aufmerksamkeit wie den geschriebenen gewidmet. Das Kapitel ist seinem Anspruch nach mit dem Artikelblock zu vergleichen, der innerhalb des Kapitels XV der diachronen Stadtsprachenforschung zugeschrieben wurde: Bei zusammenfassender Betrachtung und Auswertung der Ergebnisse des Kapitels lassen sich Grundzüge einer Prinzipienlehre und Methodik für Regionalsprachgeschichten ableiten.

Die in der ersten Auflage an unterschiedlichen Stellen eingeordneten Artikel zur Literatursprache (alte Nr. 3; 104; 156; 159; 170) gehen in der neuen Auflage in Kapitel XVII. Grundlinien einer literarischen Sprachgeschichte des Deutschen auf. Dieses hat $\mathrm{u}$. a. den folgenden Gegenstand: das epochenspezifische Verhältnis zwischen Literatursprache und der Sprache nichtliterarischer Texte; diejenigen sprachlichen Formen, an die Literarizität pro Epoche gebunden wird; die soziale und regionale Gültigkeit literarischer Varietäten.

Das Deutsche im Sprachkontakt (so formuliert in der ersten Auflage) erscheint nunmehr als Das Deutsche im Sprachenkontakt in zwei Kapiteln mit den Untertiteln Systematische und soziologische Aspekte (XIX) und Aspekte der Sprachgrenzbildung des Deutschen (XX). Die Trennung ergab sich erstens aus dem Anliegen, die Vielfalt der Gesichtspunkte der einzelnen Artikel der ersten Auflage auf die systematischen (d. h. phonemischen/graphemischen, morphologischen, lexikalischen und syntaktischen) sowie die soziologischen zu beschränken und dadurch — zweitens — der Sprachgrenzbildung einen eigenen umfänglichen Darstellungsraum zu geben. Gegenstand des Kapitels XX ist also die Grenze des Deutschen zu seinen Nachbarsprachen für die gesamte Zeitspanne vom Voralthochdeutschen und Voraltniederdeutschen bis hin zu den großräumigen Veränderungen der jüngeren Vergangenheit. So weit es möglich war, werden die Grenzen und ihre Verschiebungen kartographisch veranschaulicht. Die Ausführung dieses Programms unterlag Schwierigkeiten ganz besonderen Ausmaßes: Die historischen Verhältnisse im Süden und Südwesten, schon in sich äußerst different, sind vollständig anderer Art als im gesamten Osten; diese wiederum unterscheiden sich grundsätzlich von denjenigen des Nordens; im Nordwesten verkompliziert sich das Bild noch durch die Frage, was eigentlich Deutsch und was Niederländisch ist bzw. was als solches angesehen wird. Entsprechend unterschiedlich sind die methodischen Möglichkeiten der Programmausführung; die Erkenntnismittel der Siedlungs-, Territorial-, Kirchen-, Rechts-, Wirtschafts-, Verfassungsgeschichte wie der politischen Geschichte generell waren ebenso anzuwenden wie diejenigen der historischen Namenkunde, der Sprachgeographie und der auf sprachbezogene Identifizierungen ausgerichteten Sprachbewußtseinsgeschichte.

Den Abschluß des Handbuches bildet die Deutsche Namengeschichte im Überblick (Kap. XXI). Sie deckt sich in der Anzahl der Artikel und der Formulierung ihrer Titel mit dem entsprechenden Kapitel der ersten Auflage. - Ein detailliertes Sachregister und ein Namenregister schließen das Handbuch ab.

\section{Zur Geschichte der zweiten Auflage und Danksagungen}

Nachdem sich die Notwendigkeit einer Neuauflage des Handbuches bereits in den späten acht-

ziger Jahren abzeichnete, konnten die Herausgeber langfristig planen. Sie taten dies zunächst im alten Herausgeberkreis, seit Beginn der neunziger Jahre zusammen mit Anne Betten als 
Mitherausgeberin und in enger inhaltlicher Zusammenarbeit mit den Reihenherausgebern Hugo Steger und Herbert Ernst Wiegand sowie im Zusammenspiel mit dem Verlag Walter de Gruyter, insbesondere mit Frau Dr. Brigitte Schöning.

Die Herausgeberin und die Herausgeber verstehen die zweite Auflage trotz der in Abs. 2 beschriebenen Konstanzen in Inhalt und Anlage als ein neues Werk. Damit stellte sich zwangsläufig die Frage nach der Autorschaft der einzelnen Artikel. Zu beachten, zu bedenken, gegeneinander abzuwägen und zu gewichten waren Gegebenheiten gänzlich unterschiedlicher Art: die Autorschaft in der ersten Auflage, die Wiedervereinigung Deutschlands mit der dadurch ermöglichten Gewinnung der linguistischen Kompetenz der Wissenschaftler eines ganzen (ehemaligen) Staates, die mit dem Fortschreiten der Forschung verbundenen Erwartungen der jüngeren Forschungsgeneration, schließlich auch der Tod oder die schwere Krankheit einer Anzahl von Autorinnen und Autoren der ersten Auflage. Die Herausgeberin und die Herausgeber haben gemeint, die Frage nach der Autorschaft vorwiegend im Hinblick auf die Bedürfnisse der neuen Auflage behandeln zu sollen. Sie danken an dieser Stelle allen Beteiligten für ihre Kooperationsbereitschaft. Die Autorinnen und Autoren haben sich nicht nur zur Übernahme eines oder mehrerer Artikel bereit erklärt und ihre Zusage eingehalten; sie haben sich auch einer Gemeinschaftsaufgabe gestellt, haben ihre persönlichen Schwerpunkte in die Konzeption des Handbuches integriert, haben mit den Herausgebern diskutiert und vorgeschlagene Änderungen ihrer Artikel akzeptiert oder mit Gründen abgelehnt. Daß das Handbuch bei der Fülle der Beiträge und der Unterschiedlichkeit der Inhalte doch eine weitgehende Geschlossenheit im Hinblick auf seine Theoriegrundlage, seine Terminologie wie seine Fachsprache allgemein aufweist, ist zum großen Teil das Verdienst der Autoren. - Denjenigen Kolleginnen und Kollegen, die durch Vertragsrücktritt einiger Autoren frei gewordene Artikel übernommen haben, stand oft eine außerordentlich kurze Bearbeitungszeit zur Verfügung; ihnen sei besonders herzlich gedankt.

Die Hoffnung der Herausgeber, die zweite Auflage des Handbuches zügiger realisieren zu können als die erste, hat getrogen. Der Grund hierfür liegt sicher zum Teil in der Umfangserweiterung. Es kann aber auch nicht verschwiegen werden, daß die Zeitspanne zwischen Vertragsabschluß und Abgabe der Artikel in vielen Fällen länger wurde. Die Herausgeber bringen dies (auch) in Zusammenhang mit der unbezweifelbaren Tatsache, daß die Belastungen der Hochschullehrer in den Massenfächern sich in den letzten Jahrzehnten zunehmend gesteigert haben. Sie haben zu einer schleichenden Verdrängung der Forschung aus der konstitutionell durch die Verbindung von Forschung und Lehre gekennzeichneten mitteleuropäischen Universität geführt. Daß diese Hälftung der Infrastruktur schon in wenigen Jahren dazu führen wird, daß Vorhaben wie das Handbuch Sprachgeschichte nicht mehr an den Universitäten realisiert werden können und daß gleichzeitig ein Niedergang der Lehre und damit der wissenschaftlichen Ausbildung der Nachwuchsgenerationen eintreten wird, muß auch hier zu sagen erlaubt sein.

Es mag u. a. hiermit zusammenhängen, daß in der zweiten Auflage wesentlich mehr Artikel nicht realisiert werden konnten als in der ersten. Diese waren: Deutsche Sprachgeschichte und Geschichte der Mathematik (für Kap. I), Terminologiebildung in der Sprachgeschichtsforschung (für III), Die Geschichte der Metaphorik seit dem 17. Jahrhundert (für XIII), Fachund bildungssprachliche Jargons seit der Mitte des 20. Jahrhunderts, Norm und Lizenz in der deutschen Standardsprache seit der Mitte des 20. Jahrhunderts, Die deutsche Sprache an der Schwelle zum dritten Jahrtausend (jeweils für XIV), Aspekte einer Sprachgeschichte des Schwäbischen (für XVII); möglicherweise kommen weitere hinzu.

Die Herausgeber der Reihe Handbücher zur Sprach- und Kommunikationswissenschaft, Hugo Steger und Herbert Ernst Wiegand, haben die Planung und Vorbereitung der zweiten Auflage von Sprachgeschichte mit Interesse, Engagement und positiver Kritik bis hin zu vielen Einzelratschlägen begleitet. Ihnen sei an dieser Stelle unser herzlichster Dank ausgesprochen. 
Die Einrichtung der Manuskripte für den Druck und die Anlage des Sachregisters erfolgten am Lehrstuhl für Germanistische Sprachwissenschaft an der Universität Heidelberg durch Anja Lobenstein-Reichmann und Oskar Reichmann; zusätzliche Hilfe, speziell bei der Erstellung des Namenregisters, leistete Silke Bär; ihr und Frau Lobenstein-Reichmann sei sehr herzlich gedankt. Die Kommunikation mit den Autorinnen und Autoren oblag Oskar Reichmann am Heidelberger Lehrstuhl; Frau Ursula Quoos sorgte über Jahre hinweg mit gleichbleibender Zuverlässigkeit für die Ausführung der umfangreichen Korrespondenz.

Die Herausgabe eines in der zweiten Auflage dreibändigen Handbuches zur Geschichte der deutschen Sprache und ihrer Erforschung stellt in Zeiten knapp werdender Bibliotheksetats ein verlegerisches Risiko hohen Ausmaßes dar. Die Herausgeberin und die Herausgeber danken dem Verlag für die Übernahme dieses Risikos. Die Zusammenarbeit mit Frau Dr. Brigitte Schöning und ihren Mitarbeiterinnen, vor allem Frau Susanne Rade, Frau Heike Plank und Frau Angelika Hermann, gestaltete sich reibungslos. Ihnen sei dafür herzlicher Dank gesagt.

$\begin{array}{llll}\begin{array}{l}\text { Werner Besch } \\ \text { (Bonn) }\end{array} & \begin{array}{l}\text { Anne Betten } \\ \text { (Salzburg) }\end{array} & \begin{array}{l}\text { Oskar Reichmann } \\ \text { (Heidelberg) }\end{array} & \begin{array}{l}\text { Stefan Sonderegger } \\ \text { (Zürich) }\end{array}\end{array}$


